\title{
Zdeněk Frolík
}

Invariance of $G_{\delta}$-spaces under mappings

Czechoslovak Mathematical Journal, Vol. 11 (1961), No. 2, 258-261

Persistent URL: http://dml.cz/dmlcz/100459

\section{Terms of use:}

(C) Institute of Mathematics AS CR, 1961

Institute of Mathematics of the Czech Academy of Sciences provides access to digitized documents strictly for personal use. Each copy of any part of this document must contain these Terms of use.

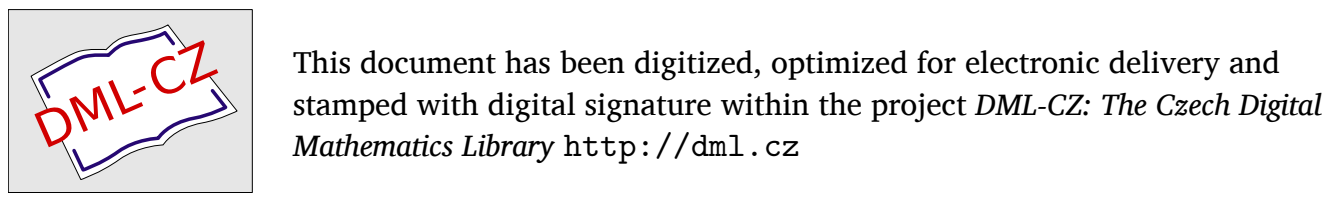




\title{
INVARIANCE OF $G_{\delta}$-SPACES UNDER MAPPINGS
}

\author{
ZDENĚK Frolík, Praha
}

(Received February 27, 1960)

\begin{abstract}
It is proved that the images and inverse images respectively, of $G_{\delta}$ spaces are also $G_{\delta}$, under certain conditions on the mapping; some related questions are also considered.
\end{abstract}

The present paper is devoted to the following two questions: Let $f$ be a continuous mapping of a space $P$ onto a space $Q$. Under what conditions on $f$ may we assert that:

(1) if $P$ is a $G_{\delta}$-space, then $Q$ is a $G_{\delta}$-space.

(2) if $Q$ is a $G_{\delta}$-space, then $P$ is a $G_{\delta}$-space.

In connection with (2) some characterisations of completely regular $G_{\delta}$-spaces are given. For example, a completely regular space $P$ is a $G_{\delta}$-space if and only if $P$ is homeomorphic with some closed subspace of the topological product of a countable family of locally compact completely regular spaces.

All spaces are assumed to be completely regular. The terminology and notation of J. KeLleY, General Topology, is used throughout. $\beta(P)$ always denotes the Čech Stone compactification of a space $P$. Let us recall that a space $P$ is said to be a $G_{\delta^{-}}$ space (or topologically complete in the sense of E. ČECH, vide [1]) if $P$ is a $G_{\delta}$-set in $\beta(P)$. The following facts are well-known (vide [1] or [2]) if a space $P$ is a $G_{\delta}$ in some of its compactifications, then $P$ is a $G_{\delta^{-}}$-space; every $G_{\delta^{-}}$-space is $G_{\delta}$ in each of its extensions (a space $R$ is an extension of a space $P$ if $P$ is a dense subspace of $R$ ).

If is well-known that a continuous image of a $G_{\delta}$-space may fail to be a $G_{\delta}$-space. Moreover, the image under a linear continuous mapping of a complete normed linear space may fail to be a $G_{\delta}$-space. Indeed, by well-known theorem, a linear continuous mapping $f$ of a complete normed linear space $P$ is open if and only if $f[P]$ is of the second category in itself.

1. Theorem. Let $f$ be an open continuous mapping of a space $P$ onto a space $Q$. If $P$ is a $G_{\delta^{-}}$space, then $Q$ is $a G_{\delta^{-}}$-space.

Proof. According to the Čech-Stone theorem, there exists a continuous mapping $F$ of $\beta(P)$ onto $\beta(Q)$ such that $f$ is the restriction of $F$ to $P$. From the fact that $f$ is open we may conclude at once that, if $U$ is an open subset of $\beta(P)$ containing $P$, then the interior of $F[U]($ in $\beta(Q))$ contains the set $Q$. Now let $P$ be a $G_{\delta}$-space. Then there 
exists a sequence $\left\{U_{n}\right\}$ of open subsets of $\beta(P)$ such that $\bigcap_{n=1}^{\infty} U_{n}=P$. Denoting by $V_{n}$ the interior of $F\left[U_{n}\right]$, we conclude as above that $V_{n} \supset Q$. Evidently $Q=\bigcap_{n=1}^{\infty} V_{n}$. Thus $Q$ is $G_{\delta}$ in $\beta(Q)$, and consequently, $Q$ is a $G_{\delta}$-space.

As an immediate consequence of the precending theorem and of the fact that a metrizable space $P$ is a $G_{\delta}$-space if and only if there exists a metric $\varphi$ for $P$ such that $(P, \varphi)$ is a complete metric space, we have at once:

2. Theorem. Let $f$ be an open continuous mapping of a complete metric space $P$ onto a metrizable space $Q$. Then there exists a metric $\psi$ for $Q$ such that $(Q, \psi)$ is a complete metric space.

It may be noticed that a continuous mapping of a $G_{\delta}$-space onto a $G_{\delta}$-space may fail to be open.

The remainder of this paper is devoted to investigations of inverse images of $G_{\delta^{-}}$ spaces under mappings of a special sort. A mapping $f$ of a space $P$ into a space $Q$ will be called closed if the images of closed sets are closed. We shall need the following

3. Lemma. Let $F$ be a continuous mapping of a space $R$ onto a space $S$. Let $P$ be a dense subspace of $R$. Suppose that the restriction $f=F / P$ of $F$ to $P$ is a closed mapping onto $Q=f[P]$. Finally, let the inverses of points (i. e. sets of the form $\left.f^{-1}[y], y \in Q\right)$ be closed in $R$. Then $F^{-1}[Q]=P$, or equivalently, $F[R-P] \subset$ $\subset S-Q$.

Proof. Suppose that there exists a point $x$ in $R-P$ such that the point $y=F(x)$ belongs to $Q$. Put $K=f^{-1}[y]$. $R$ being a regular space, there exists a neighborhood $U$ of $x$ closed in $R$ and disjoint with $K . P$ being dense in $R$, we have $x \in \overline{U \cap P}$, and by continuity of $F$,

$$
y=F(x) \in \overline{F[U \cap P]^{S}} .
$$

Since $y \in Q$ and $F[U \cap P]=f[U \cap P] \subset Q$ we obtain at once $y \in \overline{f[U \cap P]^{P}}$; $f$ being a closed mapping and $U \cap P$ being a closed subset of $P, f[U \cap P]$ is a closed subset of $Q$. Thus the point $y$ belongs to $f[U \cap P]$. But this is impossible, since the sets $K=f^{-1}[y]$ and $U$ are disjoint. This contradiction establishes the lemma.

A mapping $f$ of a space onto a space $Q$ will be called compact provided that the inverses $f^{-1}[y], y \in Q$, are compact spaces. From the preceding lemma we deduce:

4. Theorem. Let us suppose that $f$ is a continuous, closed and compact mapping of a space $P$ onto a space $Q$. If $Q$ is a $G_{\delta}$-space, then $P$ is a $G_{\delta}$-space.

Proof. According to the Čech-Stone theorem, there exists a continuous mapping $F$ of $\beta(P)$ onto $\beta(Q)$ such that $f$ is the restriction of $F$. It is easy to see that the assumptions of the preceding lemma are fulfilled an hence that

$$
F[\beta(P)-P] \subset \beta(Q)-Q .
$$


Let us suppose that $Q$ is a $G_{\delta}$-space. There exists a sequence $\left\{U_{n}\right\}$ of open subsets of $\beta(Q)$ such that $\bigcap_{n=1}^{\infty} U_{n}=Q$. According to $(*)$, we have

$$
P=\bigcap_{n=1}^{\infty} F^{-1}\left[U_{n}\right] .
$$

Thus $P$ is $G_{\delta}$ in $\beta(P)$, and consequently, $P$ is a $G_{\delta}$-space.

5. Theorem. A space $P$ is a $G_{\delta}$-space if and only if $P$ is homeomorphic with some closed subspace of the topological product of a countable family of locally compact spaces.

Proof. Let us suppose that $P$ is a $G_{\delta}$-space. There exists a sequence $\left\{U_{n}\right\}$ of open subsets of $\beta(P)$ such that $\bigcap_{n=1}^{\infty} U_{n}=P$. Consider the topological product

$$
U=X\left\{U_{n} ; n=1,2, \ldots\right\} .
$$

For every $x$ in $P$ denote by $f(x)$ the point $\{x, x, \ldots\}$ of $U$. The mapping $f$ of $P$ to $U$ is a homeomorphism and $f[P]$ is closed in $U$. The spaces $U_{n}$ being locally compact, the necessity is proved. On the other hand, it is well-known (and it may be easily proved) that the topological product of a countable family of $G_{\delta}$-spaces is a $G_{\delta}$-space, and that every closed subspace of a $G_{\delta}$-space is a $G_{\delta}$-space. The sufficiency follows.

A continuous mapping $f$ of a space $P$ to a space $Q$ will be called non-extensible if there exists no proper extension $R$ of $P$ (that is $P \underset{\subset}{\neq} R$ and $\bar{P}=R$ ) over which $f$ may be continuously extended (in other words: if $R$ is a extension of $P$ and if $F$ is a continuous mapping of $R$ to $Q$ such that $f$ is the restriction of $F$, then $P=R$ ).

6. Theorem. A space $P$ is a $G_{\delta}$-space if and only if there exists a continuous nonextensible mapping of $P$ to a $G_{\delta^{-}}$-space.

Proof. Let us suppose that $f$ is a continuous non-extensible mapping of $P$ to a $G_{\delta}$-space $Q$. According to the Čech-Stone theorem, there exists a continuous mapping $F$ of $\beta(P)$ to $\beta(Q)$. From the non-extensibility of $f$ we obtain that

$$
F[\beta(P)-P] \subset \beta(Q)-f[P] .
$$

Now by the same argument as in the proof of theorem 4, it may be shown that $P$ is a $G_{\delta}$-space. Conversely, if $P$ is a $G_{\delta}$-space then the identity mapping of $P($ to $P$ ) is nonextensible.

It may be noticed that if $P$ is a $G_{\delta}$-space, then there exists a continuous nonextensible mapping of $P$ to the topological product of a countable family of locally compact spaces. Indeed, the mapping $f$ from the first part of the proof of theorem 5 is non-extensible.

Combining the theorems 4,5 and 6 we obtain

7. Theorem. The following properties of a space $P$ are equivalent:

(1) $P$ is a $G_{\delta}$-space. 
(2) There exists a continuous, closed and compact mapping of $P$ to a $G_{\delta}$-space.

(3) $P$ is homeomorphic with some closed subspace of the topological product of a countable family of locally compact spaces.

(4) There exists a continuous non-extensible mapping of $P$ to the topological product of a countable family of locally compact spaces.

(5) There exists a continuous, closed and compact mapping of $P$ to a topological product of a countable family of locally compact spaces.

\title{
Bibliography
}

[1] E. Čech: On Bicompact Spaces. Ann. Math., 39 (1937), 823-844.

[2] Z. Frolik: Generalizations of $G_{\delta}$-property of Complete Metric Spaces. Czech. Math. J. 10 (85), 359-379.

\section{Резюме}

\section{ИНВАРИАНТНОСТЬ $G_{\delta}$-ПРОСТРАНСТВ ПРИ ОТОБРАЖЕНИЯХ}

\author{
ЗДЕНЕК ФРОЛИК, (Zdeněk Frolík) Прага \\ (Поступило в редакцию 27/II 1960 г.)
}

Вполне регулярное пространство $P$ называется $G_{\delta}$-пространством (или топологически полным в смысле Э. Чеха), если оно является $G_{\delta}$-множеством в своем чеховском бикомпактном расширении $\beta(P)$. В статье доказываются следующие теоремы:

1. Пусть $f$ - непрерывное открытое отображение вполне регулярного пространства $P$ на вполне регулярное пространство $Q$. Если $P$ является $G_{\delta}$-пространспвом, то $Q$ также является $Q_{\delta}$-пространством.

2. Пусть $f$ - замкнутое непрерывное отображение вполне регулярного пространства $P$ на вполне регулярное пространство $Q$. Если подпространства $f^{-1}[y], y \in Q$ бикомпактны и $Q$ есть $G_{\delta}$-пространство, то $P$ тоже является $G_{\delta}$-пространством.

Далее даются некоторые эквивалентные определения вполне регулярных $G_{\delta}$-пространств. Хорошо известно, что метризуемое пространство $P$ является $G_{\delta}$-пространством тогда и только тогда, когда для некоторой метрики $\varphi$ метрическое пространство $(P, \varphi)$ полно. Из 1 в частности следует:

Если существует открытое непрерывное отображение $f$ некоторого полного метрического пространства на метризуемое пространство $Q$, то для некоторой метрики $\varphi$ метрическое пространство $(Q, \varphi)$ полно. 